\title{
CD24 Expression as a Marker for Predicting Clinical Outcome in Human Gliomas
}

\author{
Jianping Deng, Guodong Gao, Liang Wang, Tao Wang, Jia Yu, and Zhenwei Zhao \\ Department of Neurosurgery, Institute for Functional Neurosurgery PLA, TangDu Hospital, \\ Fourth Military Medical University, No. 1 Xinsi Road, Baqiao District, Xi'an 710038, China \\ Correspondence should be addressed to Zhenwei Zhao, zhao.zwtd@gmail.com
}

Received 6 November 2011; Accepted 5 December 2011

Academic Editor: Xin-yuan Guan

Copyright ( 92012 Jianping Deng et al. This is an open access article distributed under the Creative Commons Attribution License, which permits unrestricted use, distribution, and reproduction in any medium, provided the original work is properly cited.

CD24 is overexpressed in glioma cells in vitro and in vivo. However, the correlation of its expression with clinicopathological parameters of gliomas and its prognostic significance in this tumor remain largely unknown. To address this problem, $151 \mathrm{glioma}$ specimens and 10 nonneoplastic brain tissues were collected. Quantitative real-time PCR, immunochemistry assay, and Western blot analysis were carried out to investigate the expression of CD24. As per the results, CD24 was overexpressed in gliomas. Its expression levels in glioma tissues with higher grade $(P<0.001)$ and lower KPS $(P<0.001)$ were significantly higher than those with lower grade and higher KPS, respectively. Cox multifactor analysis showed that CD24 $(P=0.02)$ was an independent prognosis factor for human glioma. Our data provides convincing evidence for the first time that the overexpression of CD24 at gene and protein levels is correlated with advanced clinicopathological parameters and poor prognosis in patients with glioma.

\section{Introduction}

Glioma is the most common type of primary central nervous system tumor in adults. It is a kind of aggressive tumors with a tendency to invade the surrounding brain tissue. Despite that great progress has been made in the surgical, radioactive, and chemical antiglioma modalities, the prognosis of this tumor remains poor. Especially, glioblastoma multiforme (GBM), the most malignant as well as the most reported glioma histology, leads to a median life expectancy of only 10-12 months after diagnosis [1]. While the molecular pathology responsible for the aggressiveness of human glioma and the suboptimal response to conventional therapies is largely unknown, existing data suggest that a diversity of biological changes in glioma cells may account for the poor overall survival of patients with glioma [2]. It is hoped that a greater understanding of these molecular characteristics involved in glioma cell proliferation and survival will lead to new insights into glioma-genic mechanisms and more effective targeted therapies.

CD24 is a small, heavily glycosylated, mucin-like cell surface protein that consists of 27 amino acids, and it is attached to cell membranes by a GPI anchor [3]. It is expressed in cells of the hematopoietic system, such as Bcell precursors and neutrophils, in neuronal tissues, and in certain epithelial cells, such as keratinocytes and renal tubular epithelial cells [4]. It plays important roles in cell selection and maturation during hematopoiesis. CD24 has recently raised attention and lent substantial improvements for our understanding of tumor biology. Functionally, CD24 enhances the metastatic potential of malignant cells, because it has been identified as a ligand of P-selectin, an adhesion receptor on activated endothelial cells and platelets [5]. Several studies have reported that CD24 is broadly overexpressed on many types of tumor tissues, including erythroleukemia, B-cell lymphomas, small cell lung cancer, hepatocellular carcinoma, cholangiocarcinoma, esophageal squamous cell carcinoma, pancreatic adenocarcinoma, urothelial carcinoma, prostate carcinoma, ovarian cancer, breast cancer, and primary neuroendocrine carcinomas [6-10].

In 1999, Senner et al. [11] examined the effect of CD24 on growth and migration of gliomas in vitro and in vivo. They found the overexpression of CD24 in human GBM by immunohistochemical analysis. Their data also suggested that CD24 stimulated migration of gliomas in mice. However, the correlation of its expression with 
clinicopathological parameters of gliomas and its prognostic significance in this tumor remain largely unknown. In order to address this problem, we used quantitative real-time PCR, immunochemistry assay, and Western blot analysis to investigate the expression pattern of CD24 in glioma specimens and nonneoplastic brain tissues. Next, we analyzed the correlation between CD24 expression and the glioma stage as well as the survival of patients.

\section{Materials and Methods}

2.1. Patients and Tissue Samples. This study was approved by the Research Ethics Committee of the Institute for functional neurosurgery PLA, TangDu Hospital, Fourth Military Medical University, China. Written informed consent was obtained from all of the patients. All specimens were handled and made anonymous according to the ethical and legal standards.

Fresh glioma specimens were obtained from 151 patients who underwent surgery between February 2003 and March 2006. None of the patients had received radiotherapy or chemotherapy prior to surgery. Tumors were histopathologically classified according to the WHO classification [12]. Sixty-eight of the 151 gliomas were classified as low grade (31 pilocytic astrocytomas (WHO I) and 37 diffuse astrocytomas (WHO II)), and 83 were classified as highgrade gliomas (40 anaplasia astrocytomas (WHO III), and 43 primary glioblastomas (WHO IV)). Patient data included age, sex, date, and type of initial operation, and details of the followup. Clinical information was obtained by reviewing the medical records on radiographic images, by telephone or written correspondence, and by review of death certificate. A patient was considered to have recurrent disease if this was revealed either by magnetic resonance imaging or the occurrence of new neurologic symptoms. Parts of the specimens were fixed in $10 \%$ formaldehyde and imbedded in paraffin for histological sections. Other parts were put into liquid $\mathrm{N}_{2}$ for $10 \mathrm{~min}$, then into a $-70^{\circ} \mathrm{C}$ ultrafreezer for mRNA and protein isolation. Ten nonneoplastic brain tissues from 10 patients with intractable epilepsy were served as the control.

For the analysis of survival and followup, the date of surgery was used to represent the beginning of the followup period. All the patients who died from diseases other than glioma or from unexpected events were excluded from the case collection. Followups were terminated until March 18, 2011. Treatment modalities after relapse were given according to a uniform guideline.

2.2. Quantitative Real-Time PCR. Quantitative real-time PCR (QRT-PCR) was performed in a LightCycler (Roche Diagnostics, Indianapolis, IN) as described previously. PCR was done with the following primers: $\mathrm{CD} 24$ forward primer $5^{\prime}$-TGC TCC TAC CCA CGC AGA TT-3' and reverse primer $5^{\prime}$-GGC CAA CCC AGA GTT GGA A-3'; and $\beta$-actin (NM_001101): sense, 5'-CTA CAA TGA GCT GCG TGT GGC-3'; antisense, 5' -CAG GTC CAG ACG CAG GAT GGC$3^{\prime}$. The region of CD24 do the selected primers amplify is the coding region of exon 2 (Genbank NM_013230). The
CD24 PCR fragment is $453 \mathrm{bp}$ long. The LightCycler analysis software was used to analyze the PCR data as described previously [13]. Targets were normalized using $\beta$-actin as internal standard.

2.3. Immunohistochemistry Assay. Immunohistochemistry was done using avidin-biotin immunoperoxidase technique as described previously. Briefly, following peroxidase blocking with $0.3 \% \mathrm{H}_{2} \mathrm{O}_{2} /$ methanol for $30 \mathrm{~min}$, specimens were blocked with phosphate-buffered saline (PBS) containing 5\% normal horse serum (Vector Laboratories Inc., Burlingame, CA, USA). All incubations with a monoclonal antibody against human CD24 (Ab-2, clone 24C02/SN3b, LAB VISION, Fremont, CA) at $1: 100$ dilution were carried out overnight at $4^{\circ} \mathrm{C}$. Then the specimens were briefly washed in PBS and incubated at room temperature with the anti-mouse antibody and avidin-biotin peroxidase (Vector Laboratories Inc., Burlingame, CA, USA). The specimens were then washed in PBS and color-developed by diaminobenzidine solution (Dako Corporation, Carpinteria, CA, USA). After washing with water, specimens were counterstained with Meyer's hematoxylin (Sigma Chemical Co., St Louis, MO, USA). Nonneoplastic brain tissues were used as control tissues and nonimmune IgG was also used as negative control antibody for immunohistochemical staining.

Immunostaining for $\mathrm{CD} 24$ protein expression in glioma and nonneoplastic brain tissues was quantitatively analyzed with the AxioVision Rel.4.6 computerized image analysis system assisted with the automatic measurement program (Carl Zeiss, Oberkochen, Germany). Briefly, to assess the Mean Optical Density (MOD), which represents the strength of staining signals by measuring per positive pixels, we evaluated the stained sections at 200 magnification and ten representative staining fields of each section were analyzed. The scores were evaluated two independent experienced pathologists, who were blinded to the clinicopathologic parameters and clinical outcomes of the patients. An immunoreactivity score system was applied as described previously [14]. The extensional standard was (1) the number of positively stained cells $<5 \%$ scored $0 ; 6-25 \%$ scored $1 ; 26-50 \%$ scored $2 ; 51-75 \%$ scored $3 ;>75 \%$ scored 4; (2) intensity of stain: colorless scored 0 ; pallide-flavens scored 1; yellow scored 2; brown scored 3. Multiply (1) and (2). The staining score was stratified as-(0 score, absent), + (1-4 score, weak), ++ (5-8 score, moderate), and +++ (912 score, strong) according to the proportion and intensity of positively stained cancer cells. Specimens were rescored if difference of scores from two pathologists was $>3$.

2.4. Western Blot Analysis. Glioma and nonneoplastic brain tissues were homogenized in lysis buffer (PBS, $1 \%$ Nonidet P-40 (NP-40), $0.5 \%$ sodium deoxycholate, $0.1 \%$ sodium dodecyl sulfate (SDS), $100 \mu \mathrm{g} / \mathrm{mL}$ aprotinin, $100 \mu \mathrm{g} / \mathrm{mL}$ phenylmethylsulfonyl fluoride (PMSF), sodium orthovanadate) at $4{ }^{\circ} \mathrm{C}$ throughout all procedures and sonicated for $70 \mathrm{~s}$, then add $300 \mu \mathrm{g}$ PMSF per gram of tissue and incubate on ice for $30 \mathrm{~min}$, followed by centrifugation at $15,000 \mathrm{rpm}$ for $20 \mathrm{~min}$ at $4^{\circ} \mathrm{C}$. The protein content was determined according to Bradford's method (Bradford 1976), with 


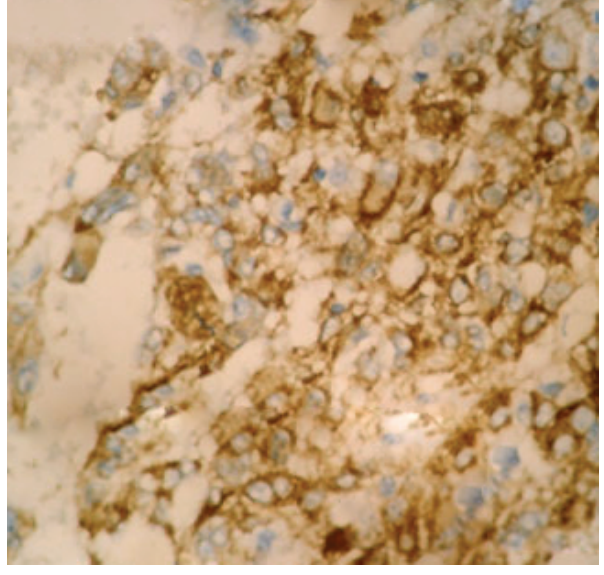

(a)

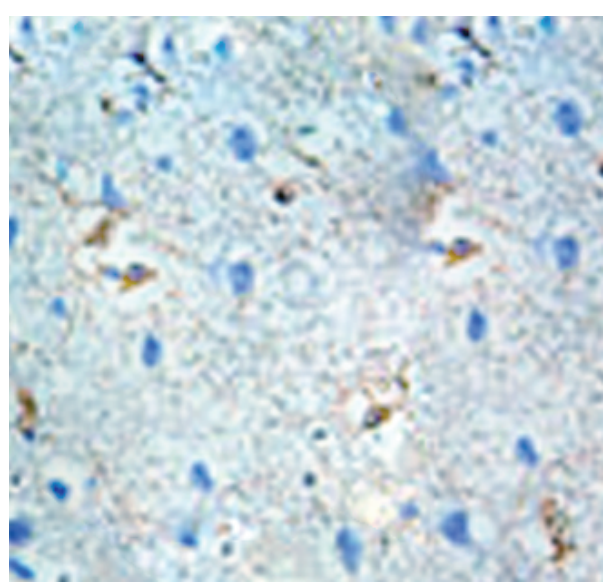

(b)

FIGURE 1: Immunohistochemical staining of CD24 protein in tumor cells of high-grade glioma (a) and nonneoplastic brain (b) tissues (original magnification $\times 400$ ). Staining for this antigen is described in Section 2. Positive staining of CD24 is seen in the cytoplasm and membrane of tumor cells in gliomas, whereas the nonneoplastic brain tissues showed no expression of CD24.

bovine serum albumin used as a standard. Protein samples $(30 \mu \mathrm{g})$ were boiled with $2 \times$ sample buffer containing $5 \% \beta$-mercaptoethanol for $5 \mathrm{~min}$, separated by size on $15 \%$ polyacrylamide gel under SDS denaturing conditions, and transferred to a nitrocellulose membrane at $90 \mathrm{~V}$ for $2 \mathrm{~h}$. The separated proteins were electrotransferred to a Hybond enhanced chemiluminescence nitrocellulose membrane (Amersham Pharmacia Biotech, Buckinghamshire, UK). Nonspecific binding was blocked by incubation in block buffer ( $5 \%$ nonfat dry milk, $0.05 \%$ Tween- $20,1 \times$ trisCl-buffered saline) overnight at $4^{\circ} \mathrm{C}$. The membranes were hybridized with mouse monoclonal antibody recognizing CD24 (Ab-1; clone SN3; Neomarkers), then incubated with a horseradish peroxidase-labeled goat anti-mouse IgG $(1: 500)$. The bound secondary antibody was detected by enhanced chemiluminescence (Amersham Life Science, Little Chalfont, UK). Housekeeping protein $\beta$-actin (A5316; clone AC-74; Sigma) was used as a loading control. Positive immunoreactive bands were quantified densitometrically (Leica Q500IW image analysis system) and expressed as ratio of CD24 to $\beta$-actin in optical density units.

2.5. Statistical Analysis. All computations were carried out using the software of SPSS version13.0 for Windows (SPSS Inc, IL, USA). The rank sum test was used to analyze the ranked data. The measurement data were analyzed by oneway ANOVA. Randomized block design ANOVA was used to analyze the statistical difference among different tissue types. The associations between protein expression and different clinical parameters were evaluated using Fisher's exact test or $\chi^{2}$ test. The cumulative survival after tumor removal was calculated according to Kaplan-Meier, with log-rank test. Multivariate survival analysis was performed on all the parameters that were found to be significant on univariate analysis by the Cox proportional hazard regression model. Differences were considered statistically significant when $P$ was less than 0.05 .

\section{Results}

3.1. Immunohistochemical Expression of CD24 Protein in Glioma Tissues and Its Correlation with Survival of Patients. CD24 expression was studied in a total of 151 glioma specimens of which 68 were low grade glioma (grade I and II) and 83 were high grade (grade III and IV). About 10 specimens taken from nonneoplastic brain tissues served as control group. Based on the immunohistochemistry analysis, CD24 was expressed in the cytoplasm and membrane of tumor cells in gliomas (Figure 1(a)), whereas the nonneoplastic brain tissues showed no expression of CD24 (Figure 1(b)). Among the glioma specimens, $110(72.8 \%)$ glioma specimens were positively stained, and $41(27.2 \%)$ glioma specimens were negatively stained. We also found a significant difference of CD24 expression between glioma and nonneoplastic brain tissues $(P<0.001)$.

Table 1 summarizes the association of CD24 immunostaining with the clinicopathological parameters of the gliomas. CD24 expression was not significantly affected by the gender and age (both $P>0.05$ ) of the patients. In contrast, CD24 expression was the closely correlated with WHO grade, as well as Karnofsky performance Status (KPS). The positive expression rates of CD24 in glioma tissues with grades III $\sim$ IV and KPS $<80$ were significantly higher than those with grades I $\sim$ II (Table 1; $P<0.01$ ) and KPS $\geq 80$ (Table 1; $P<0.01$ ), respectively.

We reviewed clinical information of these CD24-positive or -negative glioma patients. During the follow-up period, 118 of the 151 glioma patients $(78.1 \%)$ had died (23 from the CD24-negative group and 95 from the CD24positive group). In univariate survival analysis, we used the Kaplan-Meier method to calculate the cumulative survival curve and the differences in survival were accessed by the log-rank method. The conventional prognostic parameters including WHO grade and KPS reached significance for the overall survival. Patients with CD24-positive tumors had 


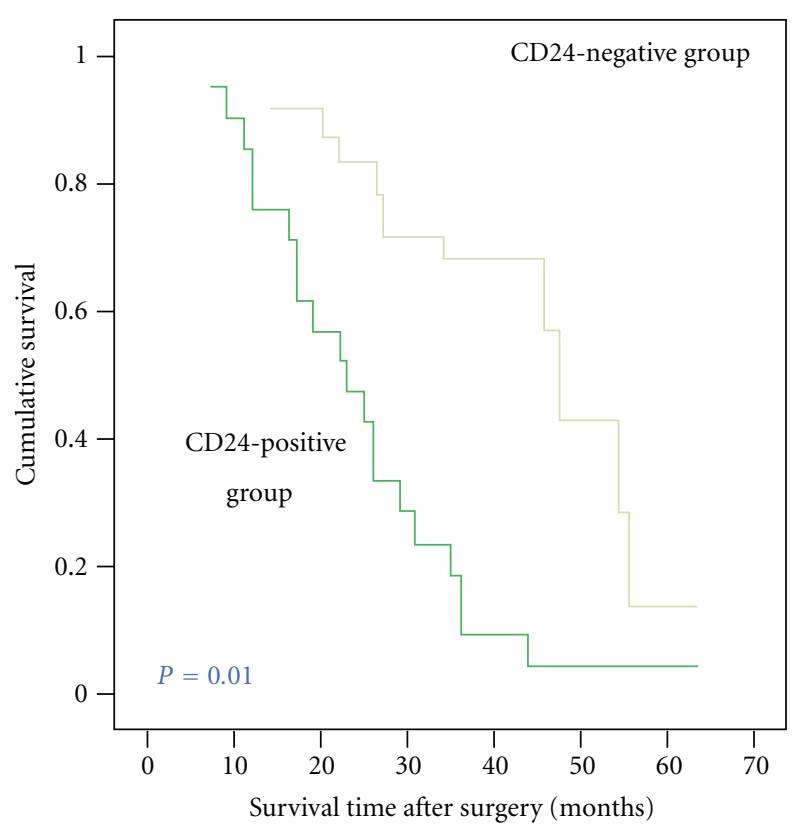

(a)

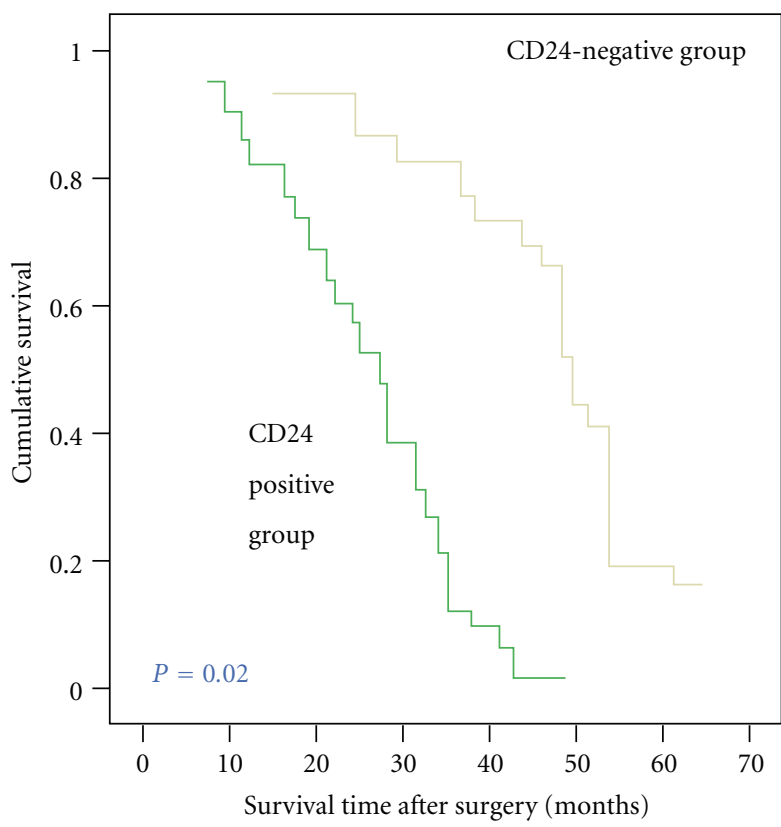

(b)

Figure 2: Postoperative survival curves for patterns of patients with glioma and CD24 expression. (a) Kaplan-Meier postoperative survival curve for patterns of patients with glioma and CD24 expression. Unadjusted RR of CD24-negative and -positive groups were 1.0 and 0.02 , respectively $(P=0.01)$. (b) Cox proportional hazards model after adjusting for age, gender, and grade. CD24 might be an independent predictor of survival, without consideration of age, gender, or grade. Adjusted RR of CD24-negative and positive groups were 1.0 and 0.04, respectively $(P=0.02)$.

TABLE 1: CD24 expression in human glioma tissues with different clinical-pathological features.

\begin{tabular}{lcccc}
\hline $\begin{array}{l}\text { Clinicopathological } \\
\text { features }\end{array}$ & \multirow{2}{*}{ No. of cases } & \multicolumn{2}{c}{ CD24 $(n)$} & \multirow{2}{*}{ Negative } \\
Positive & \\
\hline WHO grade & & & & \\
$\quad$ I & 31 & 16 & 15 & \\
II & 37 & 12 & 25 & $<0.01$ \\
III & 40 & 8 & 32 & \\
IV & 43 & 5 & 38 & \\
Age & & & & \\
$<55$ & 81 & 20 & 61 & NS \\
$\quad \geq 55$ & 70 & 21 & 49 & \\
Gender & & & & \\
$\quad$ Male & 78 & 25 & 53 & NS \\
Female & 73 & 16 & 57 & \\
KPS & & & & \\
$\quad \geq 80$ & 66 & 31 & 35 & $<0.01$ \\
$\quad<80$ & 85 & 10 & 75 & \\
\hline
\end{tabular}

a significant shorter survival than those with CD24-negative tumors $(P=0.01$; Figure $2(\mathrm{a}))$. The median survival times of patients with positive and negative expression of CD24 were $13.6 \pm 2.0$ months and $22.8 \pm 1.3$ months (log rank test: $P=0.01)$.
TABLE 2: Statistics of CD24 mRNA levels in glioma.

\begin{tabular}{lccc}
\hline & No. of cases & CD24 mean (SD) & $P$ \\
\hline $\begin{array}{l}\text { Tissue type } \\
\text { Control }\end{array}$ & 10 & $0.065(0.087)$ & $<0.001$ \\
Glioma & 151 & $2.386(0.230)$ & \\
WHO grade & & & \\
I & 31 & $0.873(0.101)$ & \\
II & 37 & $1.552(0.113)$ & $<0.001$ \\
III & 40 & $3.021(0.220)$ & \\
IV & 43 & $3.604(0.378)$ & \\
KPS & & & \\
$\geq 80$ & 66 & $1.328(0.162)$ & $<0.001$ \\
$<80$ & 85 & $3.208(0.331)$ & \\
\hline
\end{tabular}

Figure 2(b) shows the cumulative survival curve of patients with glioma and CD24 expression after adjusting for age, gender, WHO grade, and KPS. By multivariate analysis, the overexpression of $\mathrm{CD} 24(P=0.02)$ was a significant and independent prognostic indicator for patients with glioma besides age, WHO grade, and KPS. The Cox proportional hazards model showed that higher CD24 expression was associated with poor overall survival.

3.2. Western Blot Analysis of CD24 Protein Expression in Glioma Tissues with Different WHO Grades. Figure 3 shows 


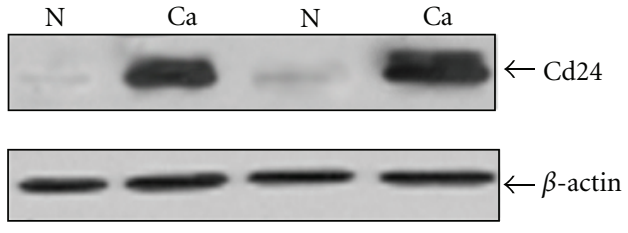

(a)

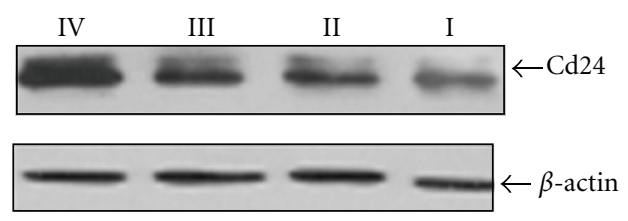

(b)

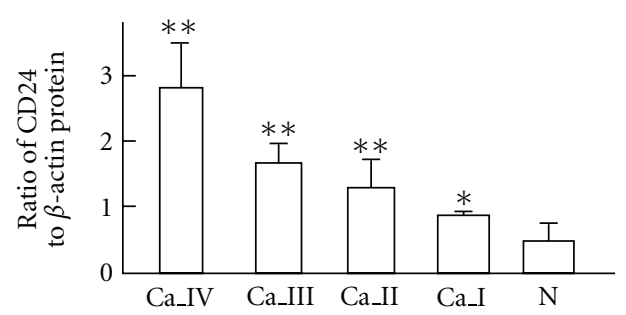

(c)

FIGURE 3: Expression of CD24 protein in glioma and nonneoplastic brain tissues by Western blot analysis. (a) CD24 expression levels in glioma and nonneoplastic brain tissues. (b) CD24 expression levels in glioma with different WHO grades. (c) CD24 expression levels in nonneoplastic brain tissues and glioma with different WHO grades. "N" refers to nonneoplastic brain tissues; "Ca" refers to glioma tissues; "Ca_ I" "Ca_ IV" refer to glioma tissues with WHO grade I IV. $\beta$-actin was used as a control for equal protein loading. Values are means \pm SD. "*”, $P<0.05$, comparison with nonneoplastic brain tissues; “**”, $P<0.01$, comparison with nonneoplastic brain tissues.

the results of quantitative analysis of $\mathrm{CD} 24$ protein expression levels detected by Western blot. We found that CD24 protein expression tended to increase from the nonneoplastic brain tissues to the glioma tissues (Figures $3(\mathrm{a})$ and $3(\mathrm{c})$ ). We also investigated whether the expression of CD24 correlated with the WHO grade. CD24 expression was lowest in grade I and highest in grade IV (Figures 3(b) and 3(c)). This result agreed with the findings of the immunohistochemistry analysis and indicated a close correlation of CD24 protein expression with WHO grade.

3.3. Quantitative Real-Time PCR Analysis of CD24 Gene Expression in Gliomas. Table 2 summarized the results of quantitative real-time PCR analysis of CD24 gene expression in gliomas with various clinicopathological features. There was a conspicuous increase in the expression of CD24 mRNA from the control nonneoplastic brain tissues to glioma tissues $(P<0.001)$. We further analyzed the expression of CD24 mRNA based on KPS and WHO grade. Interestingly, CD24 mRNA expression increased in the patients whose KPS lower than $80(P<0.001)$ and also increased with advancement of WHO grades I to IV $(P<0.001)$.

\section{Discussion}

In the present study, we examined the expression of CD24 in 151 cases of human glioma and compared its expression with tumor grade and survival rates of patients. Our data demonstrated that CD24 protein was overexpressed in gliomas compared to nonneoplastic brain tissues. CD24 mRNA expression in glioma tissues was also significantly higher than that in the nonneoplastic brain tissues. In addition, an increased trend of both CD24 protein level and mRNA level from WHO grade I to WHO grade IV glioma has been found. These results suggest that the transcriptional repression of human CD24 might participate in the carcinogenesis and progression of glioma. CD24 may have an important role during the genesis or progression of glioma. Moreover, the expression of CD24 in gliomas was a novel prognostic factor in gliomas that retained its prognostic impact on overall survival even in a multivariate analysis. To our best knowledge, this is the first study on the clinical and prognostic significance of CD24 expression in human gliomas.

CD24, first identified in 1978 when Springer produced xenogenic rat antimouse antibodies M1/69 and M1/75, was called as heat stable antigen due to its heat resistance [15]. In 1990, the human CD24 gene was identified. Human CD24 is located on chromosome 6q21, as determined by in situ hybridization [16]. The primary structure of human CD24 shows multiple $\mathrm{N}$ - or O-glycosylation sites. The human CD24 molecule has additional serine and threonine residues, rendering the molecule like a mucin. CD24 protein isolated from different tissues or cell types has different molecular weights, ranging from 20 to $70 \mathrm{kd}$, demonstrating that the glycosylation of heat-stable antigen and CD24 is highly variable and cell-type dependent [17]. It has been identified as a ligand for P-selectin and the cell adhesion molecule L1CAM. CD24 is able to support the adhesion of neutrophils or monocytes to activated endothelial cells or thrombinactivated platelets, both of which express P-selectin. CD24expressing tumor cells can disseminate more readily through their capacities to form thrombi with activated platelets or adhere to endothelial cells in the bloodstream. The interaction of tumor cells with P-selectin via CD24 may be an important adhesion pathway in cancer metastasis [18, 19]. Furthermore, CD24 expression indirectly stimulates cell adhesion to fibronectin, collagen I, collagen IV, and laminin through activation of a3b1 and a $4 \mathrm{~b} 1$ integrin activities. CD24 is regulated by Ral GTPases, suggesting that CD24 and other potential transcriptional targets of Ral signaling may mediate important functional aspects of the members of this GTPase subfamily that are emerging as central players in cancer $[20,21]$.

Accumulating evidence indicates that CD24 is an important marker for cancer diagnosis and prognosis. For various metastatic tumors, most studies have reported that the overexpression of CD24 protein might contribute to metastasis and by consequence poor prognosis. In non-small-cell lung cancer, CD24 expression is an independent marker for the overall survival of cancer patients [22]. In breast cancer, CD24 expression is significantly higher in invasive 
carcinoma than in precancerous lesions. Cell-surface and cytoplasmic expression of CD24 correlates to poor prognosis, histology grades, tumor sizes, and lymph node positivity [23]. In addition, CD24 is a marker of shorter survival for patients with intrahepatic cholangiocarcinoma [24]. Overexpression of CD24 in hepatocellular carcinoma was associated with high invasiveness and metastatic potential, high tumor proliferation status, and activation of the $\mathrm{Wnt} / \beta$ catenin pathway. CD24 may be a novel predictor for poor prognosis of HCC patients after surgery [25]. Moreover, in esophageal squamous cell carcinoma, CD24 expression correlates with tumor lymph node metastasis, tumor grade, and survival time [8]. Similar observations were found in many types of cancer, including ductal carcinoma of the pancreas, cholangiocarcinoma, urothelial carcinoma, ovarian cancer, and prostate cancer [7-10]. Furthermore, it has been demonstrated that small-interfering RNA silencing of CD24 expression in tumor cell had a direct effect on tumor cell proliferation and survival in tissue culture. For example, Overdevest et al. manipulated CD24 expression in human bladder cancer cell lines using shRNA depletion, cDNA overexpression, and FACS selection. CD24 was found necessary for subsequent development of lung metastases. They next treated clinically detectable lung metastases in mice with anti-CD24 antibody and observed reduced tumor growth and prolonged survival. These findings suggest that CD24 is a lynchpin of metastatic progression and a promising therapeutic target for antimetastatic therapy [4]. Similar observations were found by Sagiv and Arber, who demonstrated that the anti-CD24 monoclonal antibody can inhibit the growth of human pancreatic cell lines in vitro by the antibody-blocking experiments. Importantly, targeted mutation of CD24 significantly reduced the size of hepatocellular carcinomas induced by the transgenic expression of hepatitis virus B genes [26].

For human glioma, an essentially nonmetastatic tumor, CD24 might promote the tumor invasion which leads to poor prognosis of patients. CD24 has been reported to be involved in B-cell development and neoplasia, in the developing brain. Using reverse transcriptase polymerase chain reaction on 13 pineal parenchymal tumors, Fèvre-Montange et al. in 2003 demonstrated that CD24 might be one of candidates for grading pineal parenchymal tumors with intermediate differentiation [27]. Senner et al. found the immunohistochemical overexpression of CD24 in human GBM cells and it also promotes invasion of glioma cells in vivo [11]. Lo et al. [28] further showed that one of the GLI1 upregulated genes, CD24, an invasion-associated gene to be required for the migratory and invasive phenotype of GBM cells. Consistent with these previous reports, our group also confirmed that CD24 expression in gene and protein levels was both increased from nonneoplastic brain tissues to glioma tissues. Its expression levels increase from grades I to IV glioma on quantitative real-time PCR, immunohistochemistry analysis, and Western blot analysis. We further analyzed the correlation of CD24 expression and survival rates of patients. Our data indicated that the survival rate of patients with CD24 staining was lower than those without it. Kaplan-Meier analysis of the survival curves showed a significantly worse overall survival for patients whose tumors had positive CD24 expression, indicating that positive CD24 expression is a marker of poor prognosis for patients with glioma. Moreover, multivariate analysis showed positive CD24 expression to be a marker of worse outcome independent of the known clinical prognostic indicators such as age, KPS, and grade. These data suggest that positive expression of CD24 is correlated with a worse outcome of patients with glioma. Thus, CD24 might be an independent predictor of survival for glioma patients.

In conclusion, our data provides convincing evidence for the first time that the overexpression of CD24 at gene and protein levels is correlated with poor outcome in patients with glioma. CD24 expression may be a novel prognostic marker for human glioma.

\section{References}

[1] W. Wick, M. Platten, and M. Weller, "New (alternative) temozolomide regimens for the treatment of glioma," NeuroOncology, vol. 11, no. 1, pp. 69-79, 2009.

[2] A. L. Rivera, C. E. Pelloski, E. Sulman, and K. Aldape, "Prognostic and predictive markers in glioma and other neuroepithelial tumors," Current Problems in Cancer, vol. 32, no. 3, pp. 97-123, 2008.

[3] C. Poncet, V. Frances, R. Gristina, C. Scheiner, J. F. Pellissier, and D. Figarella-Branger, "CD24, a glycosylphosphatidylinositol-anchored molecule, is transiently expressed during the development of human central nervous system and is a marker of human neural cell lineage tumors," Acta Neuropathologica, vol. 91, no. 4, pp. 400-408, 1996.

[4] J. B. Overdevest, S. Thomas, G. Kristiansen, D. E. Hansel, S. C. Smith, and D. Theodorescu, "CD24 offers a therapeutic target for control of bladder cancer metastasis based on a requirement for lung colonization," Cancer Research, vol. 71, no. 11, pp. 3802-3811, 2011.

[5] K. Taniuchi, I. Nishimori, and M. A. Hollingsworth, "Intracellular CD24 inhibits cell invasion by posttranscriptional regulation of BART through interaction with G3BP," Cancer Research, vol. 71, no. 3, pp. 895-905, 2011.

[6] J. H. Myung, K. A. Gajjar, R. M. Pearson, C. A. Launiere, D. T. Eddington, and S. Hong, "Direct measurements on CD24mediated rolling of human breast cancer MCF-7 cells on Eselectin," Analytical Chemistry, vol. 83, no. 3, pp. 1078-1083, 2011.

[7] M. K. Pass, G. Quintini, J. A. Zarn, S. M. Zimmermann, J. A. Sigrist, and R. A. Stahel, "The 5'-flanking region of human CD24 gene has cell-type-specific promoter activity in smallcell lung cancer," International Journal of Cancer, vol. 78, no. 4, pp. 496-502, 1998.

[8] A. Sano, H. Kato, S. Sakurai et al., "CD24 expression is a novel prognostic factor in esophageal squamous cell carcinoma," Annals of Surgical Oncology, vol. 16, no. 2, pp. 506-514, 2009.

[9] L. R. Huang and H. C. Hsu, "Cloning and expression of CD24 gene in human hepatocellular carcinoma: a potential early tumor marker gene correlates with p53 mutation and tumor differentiation," Cancer Research, vol. 55, no. 20, pp. 47174721, 1995.

[10] Y. L. Choi, S. H. Kim, Y. K. Shin et al., "Cytoplasmic CD24 expression in advanced ovarian serous borderline tumors," Gynecologic Oncology, vol. 97, no. 2, pp. 379-386, 2005. 
[11] V. Senner, A. Sturm, I. Baur, U. H. M. Schrell, L. Distel, and W. Paulus, "CD24 promotes invasion of glioma cells in vivo," Journal of Neuropathology and Experimental Neurology, vol. 58, no. 8, pp. 795-802, 1999.

[12] D. N. Louis, H. Ohgaki, O. D. Wiestler et al., "The 2007 WHO classification of tumours of the central nervous system," Acta Neuropathologica, vol. 114, no. 2, pp. 97-109, 2007.

[13] M. Nakada, K. L. Drake, S. Nakada, J. A. Niska, and M. E. Berens, "Ephrin-B3 ligand promotes glioma invasion through activation of Rac1," Cancer Research, vol. 66, no. 17, pp. 84928500, 2006.

[14] R. S. Brown and R. L. Wahl, "Overexpression of Glut-1 glucose transporter in human breast cancer: an immunohistochemical study," Cancer, vol. 72, no. 10, pp. 2979-2985, 1993.

[15] S. Shapira, A. Shapira, A. Starr et al., "An immunoconjugate of Anti-CD24 and pseudomonas exotoxin selectively kills human colorectal tumors in mice," Gastroenterology, vol. 140, no. 3, pp. 935-946, 2011.

[16] K. Horiguchi, M. Toi, S. I. Horiguchi et al., "Predictive value of CD24 and CD44 for neoadjuvant chemotherapy response and prognosis in primary breast cancer patients," Journal of Medical and Dental Sciences, vol. 57, no. 2, pp. 165-175, 2010.

[17] H. J. Kim, M. J. Kim, S. H. Ahn et al., "Different prognostic significance of CD24 and CD44 expression in breast cancer according to hormone receptor status," Breast, vol. 20, pp. 78$85,2011$.

[18] H. J. Lee, G. Choe, S. Jheon, S. W. Sung, C. T. Lee, and J. H. Chung, "CD24, a novel cancer biomarker, predicting diseasefree survival of non-small cell lung carcinomas: a retrospective study of prognostic factor analysis from the viewpoint of forthcoming (Seventh) New TNM classification," Journal of Thoracic Oncology, vol. 5, no. 5, pp. 649-657, 2010.

[19] G. Kristiansen, E. MacHado, N. Bretz et al., "Molecular and clinical dissection of CD24 antibody specificity by a comprehensive comparative analysis," Laboratory Investigation, vol. 90, no. 7, pp. 1102-1116, 2010.

[20] W. Wang, X. Wang, L. Peng et al., "CD24-dependent MAPK pathway activation is required for colorectal cancer cell proliferation," Cancer Science, vol. 101, no. 1, pp. 112-119, 2010.

[21] D. Su, H. Deng, X. Zhao et al., "Targeting CD24 for treatment of ovarian cancer by short hairpin RNA," Cytotherapy, vol. 11, no. 5, pp. 642-652, 2009.

[22] G. Kristiansen, K. Schlüns, Y. Yongwei, C. Denkert, M. Dietel, and I. Petersen, "CD24 is an independent prognostic marker of survival in nonsmall cell lung cancer patients," British Journal of Cancer, vol. 88, no. 2, pp. 231-236, 2003.

[23] P. Athanassiadou, D. Grapsa, M. Gonidi, A. M. Athanassiadou, A. Tsipis, and E. Patsouris, "CD24 expression has a prognostic impact in breast carcinoma," Pathology Research and Practice, vol. 205, no. 8, pp. 524-533, 2009.

[24] M. C. Su, C. Hsu, H. L. Kao, and Y. M. Jeng, "CD24 expression is a prognostic factor in intrahepatic cholangiocarcinoma," Cancer Letters, vol. 235, no. 1, pp. 34-39, 2006.

[25] X. R. Yang, Y. Xu, B. Yu et al., "CD24 is a novel predictor for poor prognosis of hepatocellular carcinoma after surgery," Clinical Cancer Research, vol. 15, no. 17, pp. 5518-5527, 2009.

[26] E. Sagiv and N. Arber, "The novel oncogene CD24 and its arising role in the carcinogenesis of the GI tract: from research to therapy," Expert Review of Gastroenterology and Hepatology, vol. 2, no. 1, pp. 125-133, 2008.
[27] M. Fèvre-Montange, J. Champier, A. Szathmari et al., "Microarray analysis reveals differential gene expression patterns in tumors of the pineal region," Journal of Neuropathology and Experimental Neurology, vol. 65, no. 7, pp. 675-684, 2006.

[28] H. W. Lo, H. Zhu, X. Cao, A. Aldrich, and F. Ali-Osman, "A novel splice variant of GLI1 that promotes glioblastoma cell migration and invasion," Cancer Research, vol. 69, no. 17, pp. 6790-6798, 2009. 


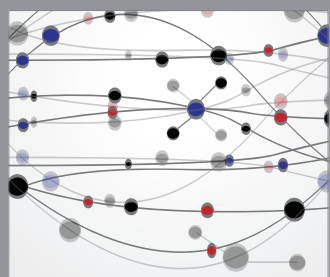

The Scientific World Journal
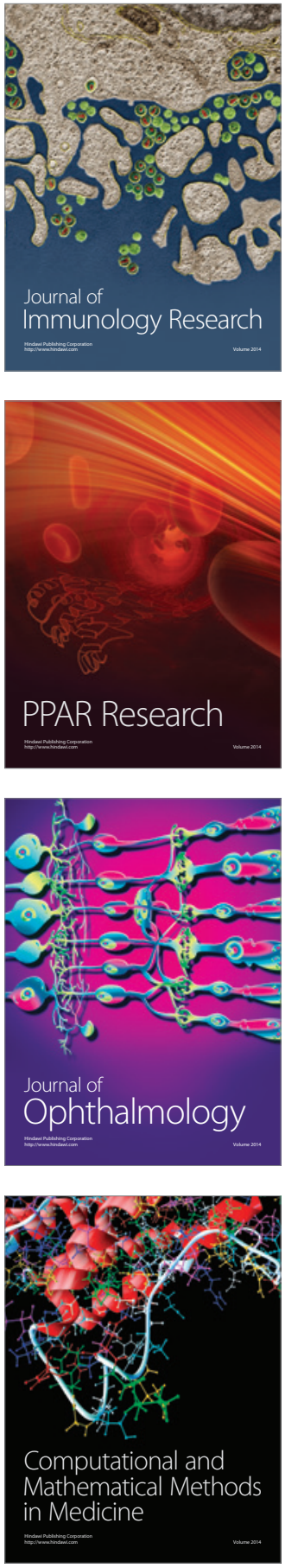

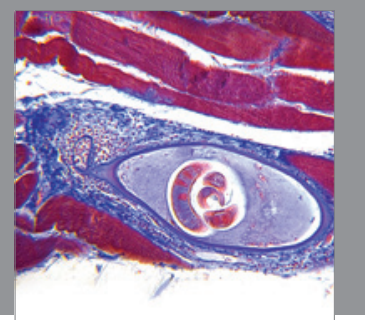

Gastroenterology

Research and Practice
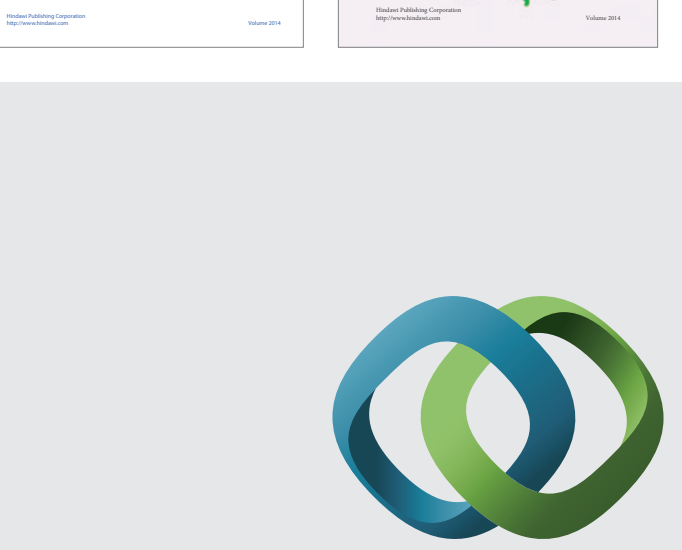

\section{Hindawi}

Submit your manuscripts at

http://www.hindawi.com
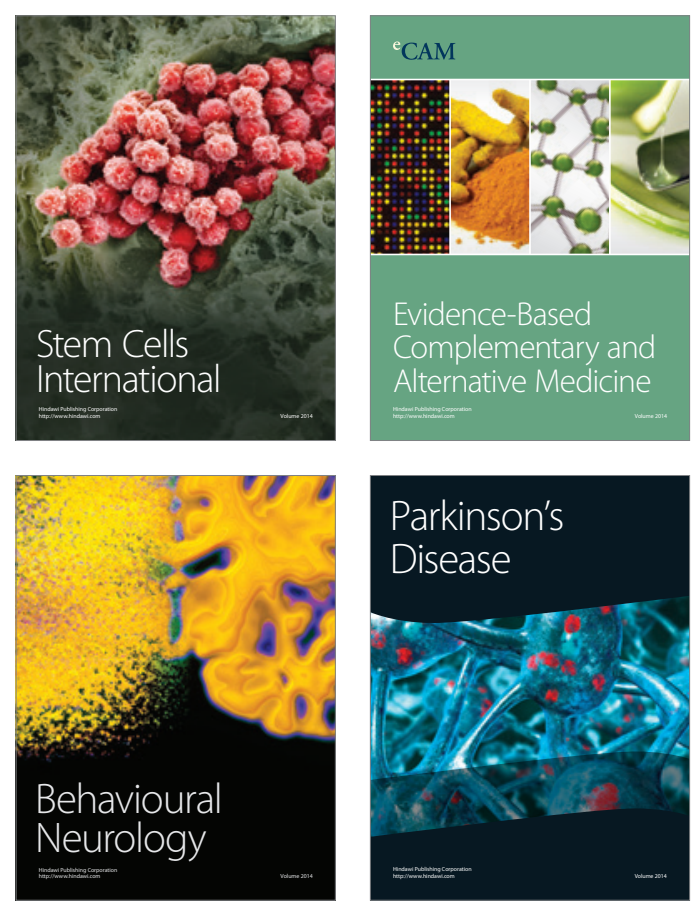

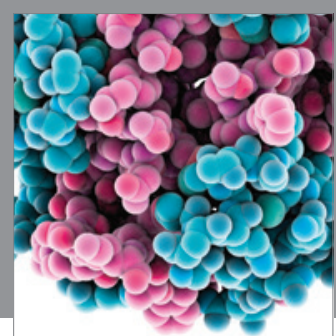

Journal of
Diabetes Research

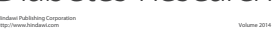

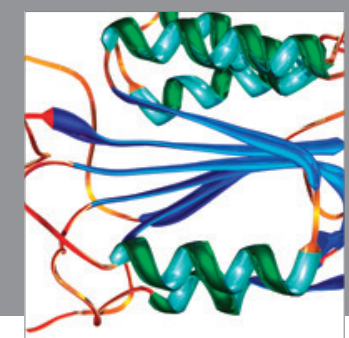

Disease Markers
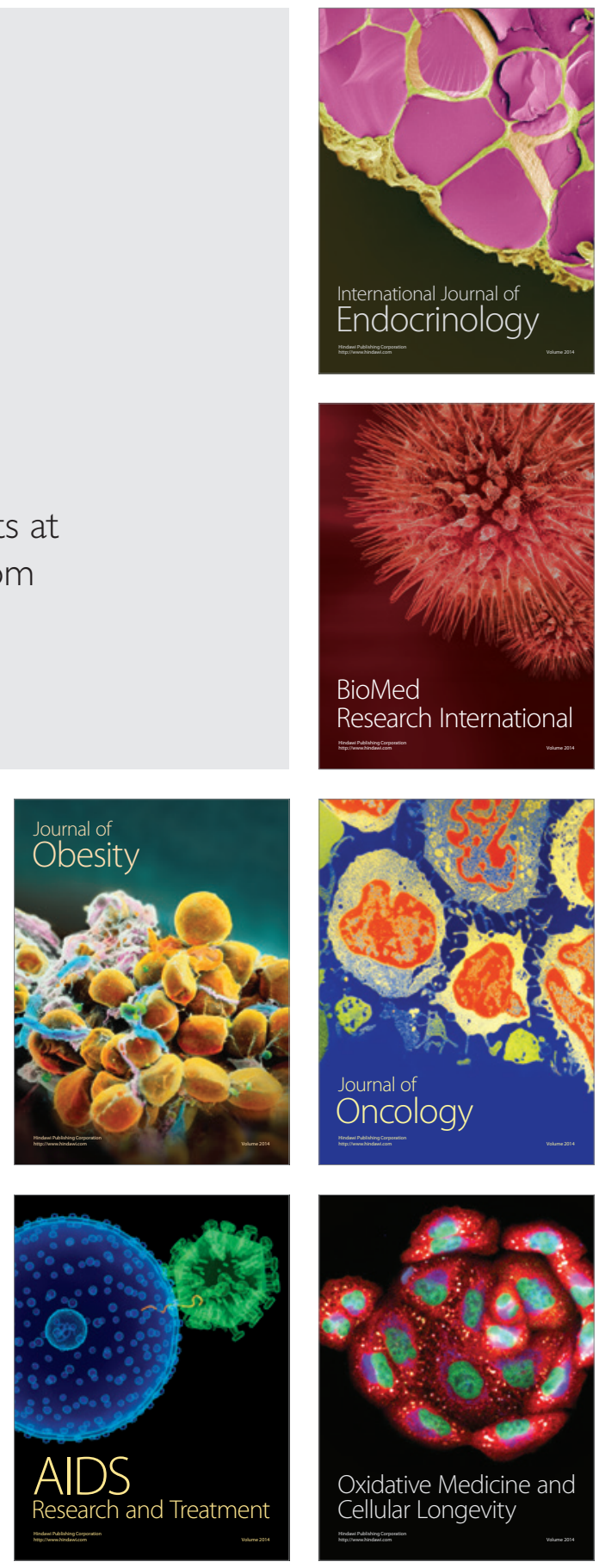